\title{
X-RAY SPECTRAL DIAGNOSTICS FOR CORONAL LOOPS IN THE ACTIVE K DWARF AB DORADUS
}

\author{
O. Vilhu ${ }^{1}$ and A. Collier Cameron ${ }^{2}$ \\ ${ }^{1}$ Observatory and Astrophysics Laboratory,University of Helsinki,Tähtitorninmäki,SF-00130 \\ Helsinki,Finland \\ ${ }^{2}$ Astronomy Centre, University of Sussex, Falmer, England BN1 9QH
}

\begin{abstract}
We discuss theoretical X-ray spectra for coronal loop models in the rapidly rotating young $\mathrm{K}$ dwarf $\mathrm{AB}$ Doradus (HD 36705), as a typical representative of active $\mathrm{X}$-ray bright stars. The loop models are based on EXOSAT and IUE observations, and further motivated by a possible connection between the observed X-ray flares and co-rotating clouds of neutral hydrogen ( $\mathrm{a}$ few/day). The resulting synthetic spectra between $0.5-7 \mathrm{keV}$ can be approximated by a linear combination of three distinct temperature components. Two components are sufficient between $0.5-2.5 \mathrm{keV}$. Below $0.1 \mathrm{keV}$ the loop spectra deviate significantly from the few component fits. To test some basic assumptions (dynamic vs. static, constant vs. variable cross-sectional area), useful constraints on the DEM(T)-distribution could be obtained with the grating-spectrometer of XMM with $10^{3}-10^{4}$ sec exposure times. The ratio of the He-type ( $O$ VII) forbidden and intercombination lines at 0.56 $\mathrm{keV}$ will provide sufficient density diagnostics, to distinguish e.g.between compact and large loops. The crystal-spectrometers of XMM and XSPECT could achieve the same but with longer $\left(10^{5} \mathrm{sec}\right)$ observing time. The strong Ly $\alpha$ line of O VIII at $0.65 \mathrm{keV}$ can be observed with the crystals in $10^{4}$ sec, and used even for rotational modulation and flare studies, and giving additional information about flows in flaring loops. At the Iron $6.7 \mathrm{keV}$ lines, where the gratings do not work, the crystals should be used together with low resolution devices, to set constraints on the hottest gas at loop summits. Our discussion applies also to several brighter cool stars but with shorter observing time (like Capella and HR 1099, which are over 3 times X-ray brighter than AB Dor).
\end{abstract}

\section{Loop Models. X-ray Flares and Cool Clouds.}

During the last years a picture has emerged in which stellar late type X-ray sources with the greatest $\mathrm{X}$-ray to optical ratios tend to have high axial rotation rates. These are either young objects, associated with regions of star formation, or else are components of close binaries with tidally locked rotation. Their X-ray spectra are usually well fitted by one or two component thin plasma models. Apart from simple scaling laws, not much is known about the structure of the emitting regions, since the heating mechanism is not known.

A good example to study stellar magnetic activity at high levels is the young $K$ dwarf $A B$ Doradus (HD $36705, \mathrm{~V}=7, \mathrm{P}=0.514 \mathrm{~d}$ ). It has been studied by e.g.Vilhu, Gustafsson and Edwardsson (1987), Collier Cameron et al.(1988), and Collier Cameron (1988). Details of the coronal loop models, based on EXOSAT observations, have been described in detail by Collier Cameron (1988). The models are constructed by solving the equations of hydrostatic equilibrium and energy balance along a static loop in a rotating reference frame, so that the effects of centrifugal forces are taken into account. The loop geometry is assumed to be that of a dipole field. The equations are solved subject to the boundary conditions that $\mathrm{Tn}_{e}^{2}(\mathrm{dT} / \mathrm{ds})^{-1}=3.9610^{28} \mathrm{~cm}^{-5}$ in the C IV resonance lines, as derived from the IUE observations, and the total heating rate $F\left(\right.$ tot) is fixed at $1.610^{8} \mathrm{erg}$ $\mathrm{cm}^{-2} \mathrm{~s}^{-1} . \mathrm{F}$ (tot) is close to the saturation value (see Vilhu,1987). 
The resulting thermally stable solutions have summit temperatures around $210^{7} \mathrm{~K}$ (compatible with the EXOSAT ME-fits) and summit heights of several stellar radii. For loops of this size the effect of centrifugal forces is important. An interesting scenario for the formation of the observed $\mathrm{H} \alpha$ clouds and their connection to large X-ray loops have been developed by Collier Cameron and Robinson (1988a,b) and Collier Cameron (1988). If an X-ray loop on AB Dor grows larger with time, its summit will eventually extend beyond the Keplerian co-rotating radius $\left(2.7 \mathrm{R}_{*}\right)$, where the centrifugal acceleration along the field direction will balance the gravity. The plasma density will then increase outwards from this point to the summit, causing the radiative loss rate to increase. When the loss rate exceeds the heating, a temperature minimum develops at the loop summit, and the summit becomes thermally unstable.

If radiative collapse then occurs at the loop summit, we would expect to see material flowing upward along the loop ( $\mathrm{a}$ few hundreds $\mathrm{km} / \mathrm{s}$ ), following the pressure collapse at the summit. The material in the unstable region will cool until hydrogen recombines, as in solar prominence formation. Actually new clouds of neutral hydrogen, trapped at fixed longitudes, are observed to form at a rate a few/ day. Three strong X-ray flares have been observed in AB Dor (Collier Cameron et al.1988;Pakull,1981;see also Vilhu and Linsky,1987) giving a similar frequency. The long risetimes of flares point to large flaring regions. Once the cloud has formed, it does not dissociate itself from the parent loop immediately. It moves radially outward (in the co-rotating reference frame) and appears to be stretching the confining loop outwards as it goes. We suspect that the cloud finally escapes from the stellar field when the field reconnects below the cloud. The resulting process might resemble a large 2-ribbon flare.

\section{Synthetic X-ray Spectra}

Each loop model solution specifies the run of temperature and electron density along the loop, whose global shape follows a line of dipole field aligned with the stellar rotation axis. Thermally stable models, with uniform cross-section, have a linear differential emission measure DEM(T) vs. $T$, and these were used to compute synthetic X-ray spectra over the energy range $0.05-7 \mathrm{keV}$, using the thin plasma code developed by Raymond (1987). The specific model, used in the present discussion, has base-to-summit length $L=3.110^{11} \mathrm{~cm}$, so that the summit lies at $3 R_{*}$ above the photosphere, and the footpoints occupy the entire surface of the star.

The spectra shown give the expected count rates at Earth for two spectral resolutions. These count rates can then be compared with the performances ( $5 \sigma$-detections) of the Grating and Crystal spectrometers in the Report of the Instrument Working Group of XMM (Briel et al.,1987), although the resolution of the crystal is still higher (comparable to the thermal broadenings of $10^{7} \mathrm{~K}$ plasma: $0.3 \mathrm{eV}$ at $0.57 \mathrm{keV}$ and $2 \mathrm{eV}$ at $6.7 \mathrm{keV}$ ). We assume that the OXS-spectrometer of the Danish XSPECT-telescope on board of the future Soviet observatory SPECTRUM-X will have a roughly similar performance than the XMM crystal (Schnopper,1987 and 1988).

Figures 1 - 3 show the synthetic spectra (lower panels) starting from the Oxygen lines at $0.56 \mathrm{keV}$,through the Silicon and Sulphur lines (at 1.9 and $2.5 \mathrm{keV}$, respectively), up to the Iron $\mathrm{XXV}$ fluorescence $\mathrm{K} \alpha$ lines at $6.7 \mathrm{keV}$. A linear combination of three components with temperatures $10^{6.5} \mathrm{~K}, 10^{7.0} \mathrm{~K}$ and $10^{7.5} \mathrm{~K}$, and with relative emission measures $0.4,1.0$ and 0.45 , respectively, can fairly well fit the synthetic spectrum in the whole energy interval $0.5-6.8 \mathrm{keV}$. At very low energies (below $0.1 \mathrm{keV}$ ) the loop spectrum deviates significantly from the spectrum predicted by the combination of these three single temperatures. A 2T-fit is sufficient for the narrower energy range $0.5-2.5 \mathrm{keV}$, perhaps explaining the success of two component fits with the EINSTEIN and EXOSAT data. The reason is natural since the region between $0.5 \mathrm{keV}-2.5 \mathrm{keV}$ contains lines formed at temperaturec ; between 1 and 10 million Kelvin. The Iron $6.7 \mathrm{keV}$ (He4, He5 and He6) fluorescence lines peak at $\mathrm{T}=10^{7.8} \mathrm{~K}$, so that one more higher temperature (somewhat non-unique) must be added to the $2 \mathrm{~T}$-model (with $\mathrm{EM}=0.45$ ). In the energy interval $0.5 \mathrm{keV}-6.7 \mathrm{keV}$, even 
with a moderate resolution $(\mathrm{E} / \mathrm{dE}=100)$ one is able to derive useful constraints on loop models. It might not be possible to separate between different static models, but to obtain useful DEM(T)-data to distinguish static vs. dynamic loops and to set important limits for the amount of the hottest gas (loop summits) with the help of the Fe $6.7 \mathrm{keV}$ lines. An important constraint on loop models can be set when a useful estimate of the plasma density becomes available. This is related to the size of the emitting volume; at present only estimates of the total emission measure can be made. The density $\mathrm{n}_{e}$ (about $310^{10} \mathrm{~cm}^{-3}$ at the formation temperature $210^{6} \mathrm{~K}$ of the Oxygen lines) is directly related to our basic assumpions that the filling factor of the loop footpoints equals to 1 (the whole stellar surface covered by footpoints), and that the CIV emission lines determine the differential emission measure at $10^{5} \mathrm{~K}$. These assumptions can be directly tested by observing the Oxygen ( $O$ VII) lines at $0.56 \mathrm{keV}$ (Fig.4). The region contains resonance (W), intercombination (X) and forbidden (Z) lines, and in addition a weak OVI-line ( $\mathrm{Li}$. The ratio $\mathrm{W} / \mathrm{Li}$ is sensitive to temperature, while the ratio $X / Z$ is a useful density-indicator.

Figure 4 shows the effect when the loop densities are increased by factors 3 and 10 (the filling factors decreased by 3 and 10 , respectively). The ratio $X / Z$ changes by more than a factor of 2 , so that reasonably good density -estimates can be expected with the XMM- grating, using 1000 10000 sec exposure times. With the crystals, $10^{5} \mathrm{sec}$ exposure times are needed. With much better resolution $(0.3 \mathrm{eV})$, the crystals can provide additional important information about flow-velocities $(0.5 \mathrm{eV})$, predicted by dynamic loop models (compare:the thermal broadening at $\mathrm{O}$ VII is $0.1 \mathrm{eV}$ ). For line profile studies the strong near-by O VIII line at $0.65 \mathrm{keV}(19 \mathrm{~A})$ can be observed with the crystals in $10^{4} \mathrm{sec}$, and can be used even for rotational modulation and flare studies. Good densityand velocity-diagnostics can then be made for both the quiescent and flaring states of $A B$ Dor, using one day observation with the crystal at $0.56-0.65 \mathrm{keV}$.

\section{References}

Briel U. et al. 1987, The High-Throughput X-Ray Spectroscopy Mission,ESA SP-1092. Collier Cameron A.et.al. 1988,MNRAS 231,131.

Collier Cameron A. 1988,MNRAS 233,235.

Collier Cameron A. and Robinson R.D. 1988a ,MNRAS, in press.

Collier Cameron A. and Robinson R.D. 1988b, in A.K. Dupree and M.T.V. Largo (eds),Formation and Structure of Low Mass Stars, (in press).

Pakull M.W. 1981, Astr.Ap.104,33.

Raymond J.C 1987, private comm.

Schnopper H.W. 1987, Lectures given at the NATO Advanced Study Institute, Cargese,

Schnopper H.W. 1988, X-ray Astronomy: A Status Report, an invited talk given at the Annual Meeting of the Finnish Physical Society,Arkhimedes 2/1988.

Vilhu O. 1987, in J.L.Linky and R.E.Stencel (eds.), Cool Stars, Stellar Systems and the Sun,Lecture Notes in Physics, Springer-Verlag,p.110.

Vilhu O., Gustafsson B. and Edvardsson B. 1987,Ap.J.320,850.

Vilhu O. and Linsky J.L. 1987,PASP 92,1071.

\section{Figures}

- Figs.1-3 Synthetic loop spectra of AB Dor, together with the few temperature fits.

- Fig.4 The loop spectrum around the $O$ VII triplet at $0.56 \mathrm{keV}$. The dashed lines correspond to the increase of the density by factors of 3 and 10 (or decreasing the foot-point filling factor by the same amounts). 

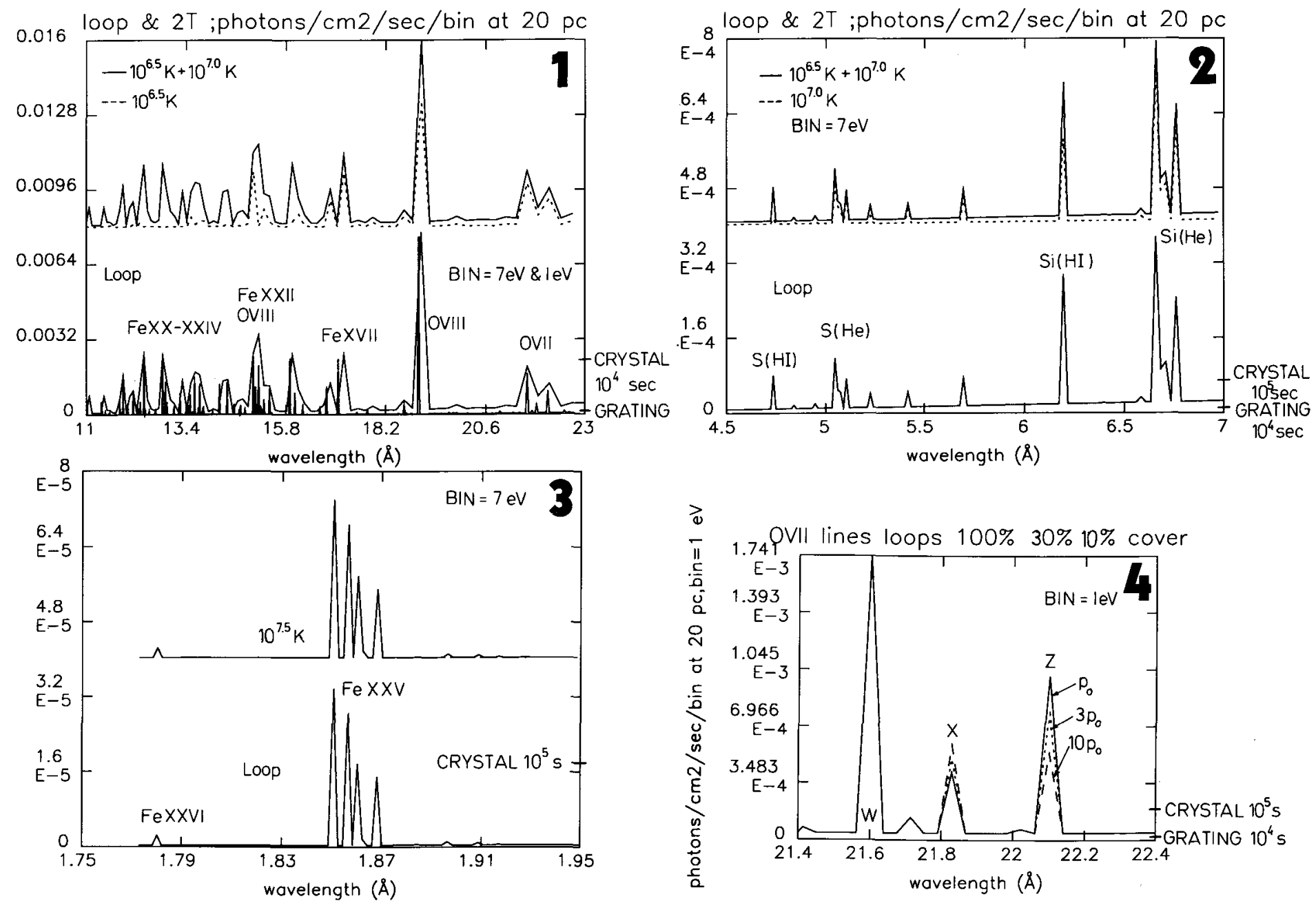